\title{
THE STARS THAT POWER THE GALACTIC CENTRE
}

D. A. Allen Anglo-Australian Observatory

A. R. Hyland Mount Stromlo \& Siding Spring Observatories

D. J. Hillier Harvard Smithsonian Center for Astrohpysics

J. A. Bailey Anglo-Australian Observatory

Using the infrared grating spectrometer FIGS on the Anglo-Australian Telescope we have mapped the inner parsec of the Galaxy. We used spectral resolutions of 1000 to observe He I $\lambda 2.058$ and Brackett $\alpha$, and 400 for complete K-band spectra.

That the region of IRS 16 comprises hot stars is apparent from the JHKL' data of Storey and Allen (1983). From our K spectra taken over a grid of positions we can separate stars with prominent $\mathrm{CO}$ absorption from those with smooth continua in the 2.3micron region. The separation, shown in Fig. 1, confirms that the blueness of the IRS 16 components is due to their earlier spectral type rather than to lower reddening. The distribution of hot stars appears more extended to the west than IRS 16 itself. Note also the blue components to IRS 10 and 5 in the northern arm.
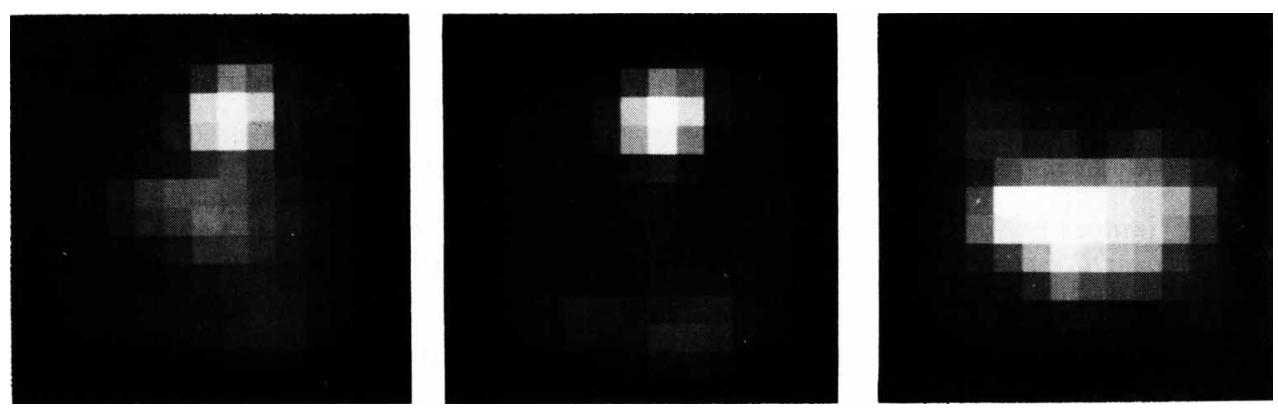

Fig. 1. Left: grey scale representations of total intensity from the 2-micron cube. The separation into regions with and without $C O$ absorption is shown at centre and right respectively. The grey scales are adjusted to suit each image. The coverage is 25 arcsec square centred on IRS 16. NE is top left, and IRS 7 is the brightest object.

Nowhere do we find lines of helium or hydrogen broader than $1100 \mathrm{~km} / \mathrm{s}$ at their base or $750 \mathrm{~km} / \mathrm{sec}$ FWHM. Fig. 2 shows a spectrum of $\mathrm{Br} \alpha$ taken in a 3.5 arcsec beam centred on IRS 16. A red wing is widely found; comparable blue wings are rare or absent. The spectrum can be compared to that of Geballe et al. (1987): we do not confirm the wide emission pedestal they reported. Fig. 3 shows the line profile in the vicinity of Sgr A*/IRS $16 \mathrm{NW}$ where the narrow component is absent. Our data are entirely consistent with a broad, redshifted line, as seen in Fig. 3, arising solely from the region of hot stars in Fig. 1(right), 
but in parts largely swamped at our spectral resolution by intense narrow components.

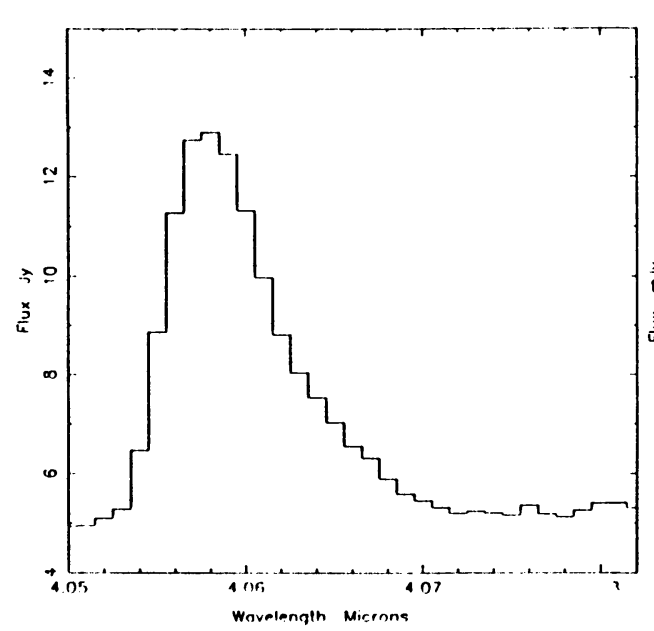

Fig. 2. Profile of $B r \alpha$ from IRS 16; 3.5 arcsec beam.

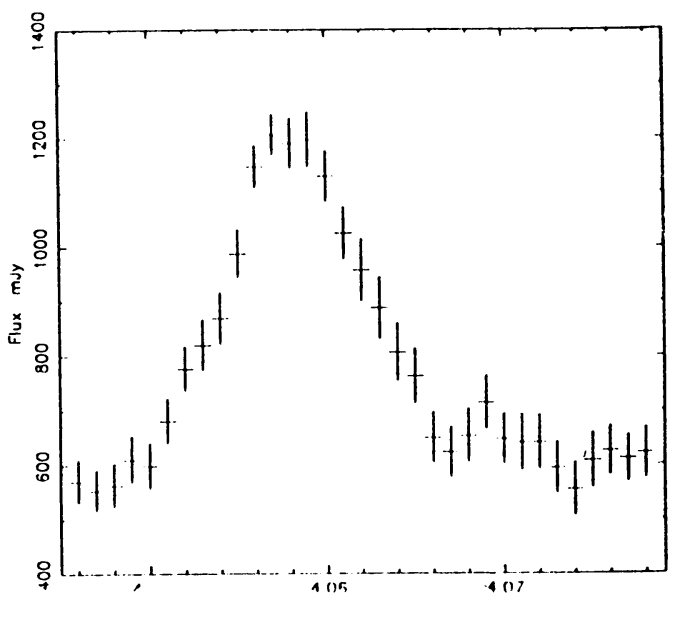

Iig. 3. Profile of $B r \alpha$ from Sgr $A^{*}+$ IRS $16 N W ; 1.4$ arcsec beam.
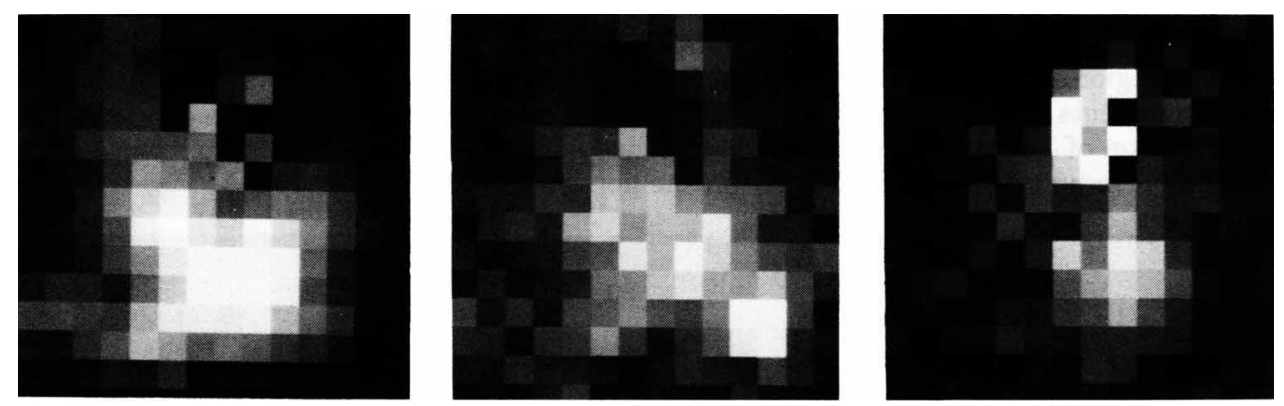

Fig. 4. Images in (left to right) $\mathrm{Br} \gamma, \mathrm{He} I \lambda 2.058$ and an unidentified line at $2.216 \mu \mathrm{m}$. These cover the same area as Fig. 1. In the process of continuum subtraction IRS 7 leaves residual noise.

In Fig. 4 we have extracted images of the principal emission lines. Most of the emission resembles the thermal radio structure (e.g. Lo and Claussen 1983). However, there is a prominent peak in He I to the south west of the centre of activity. Spectra of this region, which we associate with a point source of $\mathrm{K} \sim 10$, are given in Figs. 5 and 6. The spectra include some contamination from the underlying cusp of giant stars: $\mathrm{CO}$ absorption is seen longward of $2.3 \mu \mathrm{m}$. The extreme $\mathrm{He} \mathrm{I} / \mathrm{Br} \gamma$ ratio and the breadth of the emission lines $(750 \mathrm{~km} / \mathrm{s}$ FWHM) characterises this star as spectral type WN9, similar to HD 313846 in the Galaxy (Hutchings 1979; van der Hucht et al. 1981) and to several stars in the Large Magellanic Cloud (Walborn 1977; Stahl 1986). Assuming it to lie near the Galactic centre, the absolute $\mathrm{K}$ magnitude of -7 is also typical of the LMC stars. Any WN5-8 stars in the Galactic centre would certainly be obvious, so this is the earliest WN star in the region. The redshift, along with that of the other broad components, may reflect bulk motion of the gas from which the stars formed.

McGregor, Hyland and Hillier (1988) obtained spectra of several emission-line su- 
pergiants in the LMC which have various spectral classifications. Included were the WN9 stars HD 269445 and HD 269852. All the stars have temperatures up to $30,000 \mathrm{~K}$ and luminosities around $10^{5}-10^{6} \mathrm{~L}_{\odot}$, exactly the characteristics required of the hot stars in the Galactic centre if they are to account for virtually all the luminosity and ionization of the region (Townes et al. 1983; Adams et al. 1988). McGregor et al. found the ratio $2.058 \mu \mathrm{m}$ $\mathrm{He} \mathrm{I} / \mathrm{Br} \gamma$ to encompass that found in the components of IRS 16. The mean FWHM of the He I line in their spectra is $750 \mathrm{~km} / \mathrm{s}$.

We suggest that the central region of our Galaxy is powered by young supergiant emission OB stars together with some very late WN stars, similar to those found in the Magellanic Clouds.

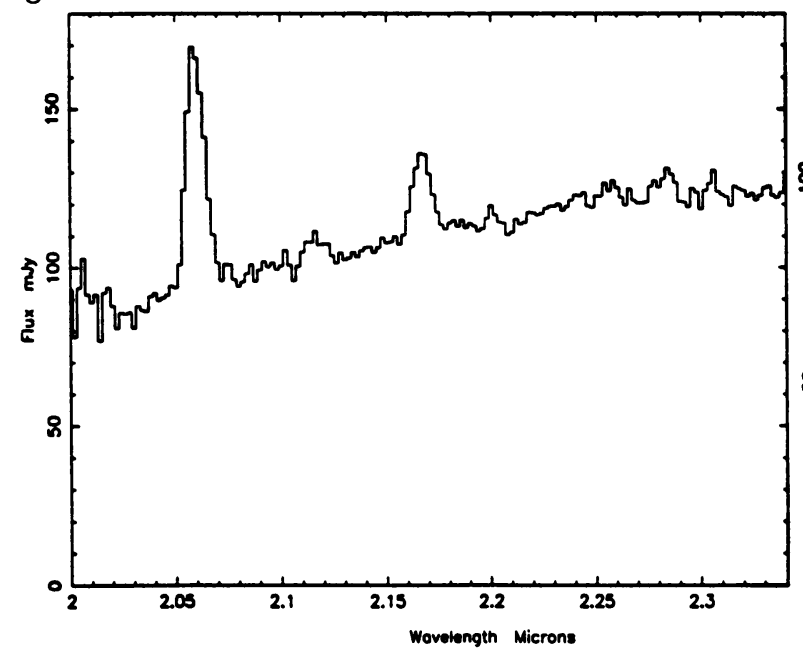

Fig. 5. Spectrum at the region of peak He I emission, uncorrected for reddening

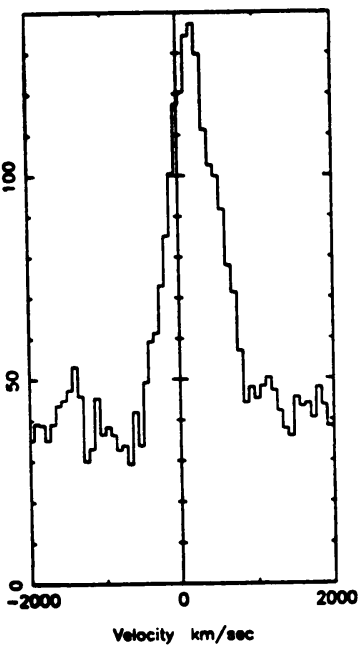

Fig. 6. Line profile of

He I emission from same star

\section{REFERENCES}

Adams, D. J., Becklin, E. E., Jameson, R. F., Longmore, A. J., Sandqvist, A. and Valentijn, E., 1988. Astrophys. J., 327, L65.

Geballe, T. R., Wade, R. Krisciunas, K., Gatley, I. and Bird, M. C., 1987. Astrophys. J., $320,562$.

van der Hucht, K. A., Conti, P. S., Lundström, I. and Stenholm, B. S., 1981. Space Sci. Rev., 28, 227.

Hutchings, J. B., 1979. Publ. astr. Soc. Pacif., 91, 361.

Lo, K. Y. and Claussen, M. J., 1983. Nature, 306, 647.

McGregor, P. J., Hillier, D. J. and Hyland, A. R., 1988. Astrophys. J., in press.

Stahl, O., 1986. Astr. Astrophys., 164, 321.

Storey, J. W. V. and Allen, D. A., 1983. Mon. Not. R. astr. Soc., 204, 1153.

Townes, C. H., Lacy, J. H., Geballe, T. R. and Hollenbach, D. J., 1983. Nature, 301, 661.

Walborn, N. R., 1977. Astrophys. J., 215, 53. 